\title{
ON A THEOREM OF GOFFMAN AND NEUGEBAUER
}

\section{A. P. BAISNAB}

1. Suppose that a function $f$ is defined in an open interval of which $I_{0}=[a, b]$ is a closed subinterval. In this paper we prove Theorem 2'1 and Theorem $3^{\prime} 2$ as two generalizations of the following theorem due to C. Goffman and C. J. Neugebauer [1].

Theorem 1'1. Suppose that (i) $f$ has an approximate derivative $f_{\mathrm{ap}}^{\prime}$ in $I_{0}$, and

(ii) $f_{\mathrm{ap}}^{\prime}(x) \geqq 0$ for all $x$ in $I_{0}$. Then $f$ is monotone increasing in $I_{0}$.

For definitions and notations used, please see S. Saks [3, p. 220]. Unilateral approximate semicontinuity of $f$ is defined in a natural way.

2. THEOREM 2'1. If (i) $f$ is approximately upper semicontinuous (a.u.s.c.) on the left, and approximately lower semicontinuous (a.l.s.c.) on the right at each point of $I_{0}$, and

(ii) Int $\{f(E)\}=\varnothing$, where $E=\left\{x: f_{\mathrm{ap}}^{+}(x) \leqq 0\right\}$, then $f$ is monotone increasing in $I_{0}$.

Proof. Let there exist two points $c, d$ in $I_{0}$, with $c<d$, such that $f(c)>f(d)$. We seek a contradiction. Since Int $\{f(E)\}=\varnothing, f(E)$ does not contain an interval, and, therefore, we can find a number $\eta$ such that $f(c)>\eta>f(d)$ and

$$
\eta \notin f(E) .
$$

We now construct a point $\xi$ in $E$ such that $\eta=f(\xi)$. This will be the desired contradiction. Let $G=\{x: f(x) \geqq \eta\}$. Then $c \in G$. Since $f$ is a.l.s.c. on the right at $c$, it follows that the set $\{x: f(x)>\eta\}$, and hence $G$ has right-density unity at $c$. Therefore, we can find a point $x_{1}$ in $G$, with $x_{1}>c$, such that

$$
\frac{\mu\left\{G \cap\left(c, x_{1}\right)\right\}}{x_{1}-c} \geqq \frac{1}{2} .
$$

Now we adopt a technique due to Goffman and Neugebăer [1], and proceed as follows.

Received by the editors March 16, 1969. 
Let $\mathcal{H C}$ denote the family of all subsets $H$ of $G$ having the following property: If $x_{1}, x_{2} \in H$, and $x_{1}<x_{2}$, then

$$
\frac{\mu\left\{G \cap\left(x_{1}, x_{2}\right)\right\}}{x_{2}-x_{1}} \geqq \frac{1}{2} .
$$

TC is not empty, since from $\left(2^{\prime} 2\right)$ it is evident that $H=\left\{c, x_{1}\right\}$ belongs to $\mathfrak{H C}$. We now partially order $\mathfrak{H C}$ by set-inclusion. It is easily verified that every chain in $\mathfrak{H C}$ has an upper bound in $\mathfrak{H}$. By Zorn's Lemma, we conclude that $\mathfrak{H C}$ has a maximal member $H_{0}$, say. Let

$$
\xi=\sup \left\{x: x \in H_{0}\right\} .
$$

If $x$ belongs to $H_{0}$, and if $x<\xi$, we shall show that

$$
\frac{\mu\{G \cap(x, \xi)\}}{\xi-x} \geqq \frac{1}{2} .
$$

From $\left(2^{\prime} 4\right)$ it follows that we can find a sequence $\left\{x_{n}\right\}$ of points of $H_{0}$ and that $x<x_{n} \leqq \xi$, and $x_{n} \rightarrow \xi_{-}$. From $\left(2^{\prime} 3\right)$ we have

$$
\frac{\mu\left\{G \cap\left(x, x_{n}\right)\right\}}{x_{n}-x} \geqq \frac{1}{2} .
$$

Taking the limit as $n \rightarrow \infty$, we have

$$
\frac{\mu\{G \cap(x, \xi)\}}{\xi-x} \geqq \frac{1}{2} .
$$

Thus $\left(2^{\prime} 5\right)$ has been established. Suppose that $\xi \notin H_{0}$. Then $\left(2^{\prime} 5\right)$ implies that $G$ has no zero left-density at $\xi$. Since $G=\{x: f(x) \geqq \eta\}$ and since $f$ is a.u.s.c. on the left at $\xi$, it follows easily that $\eta \leqq f(\xi)$. If $\xi \in H_{0}$, then $\xi \in G$ and again $\eta \leqq f(\xi)$. Suppose that $\eta<f(\xi)$ then since $f$ is a.l.s.c. on the right at $\xi$, we conclude that $G=\{x: f(x) \geqq \eta\}$ has right density 1 at $\xi$. So, we choose $x \in G$ such that $x>\xi$, and

$$
\frac{\mu\{G \cap(\xi, x)\}}{x-\xi} \geqq \frac{1}{2} .
$$

Since $H_{0}$ is maximal w.r.t. the property (2'3), and $x \notin H_{0}$, there exists $h_{0} \in H_{0}$ such that

$$
\frac{\mu\left\{G \cap\left(h_{0}, x\right)\right\}}{x-h_{0}}<\frac{1}{2} .
$$

Clearly, $h_{0}<\xi$. From $\left(2^{\prime} 5\right)$ we have 


$$
\frac{\mu\left\{G \cap\left(h_{0}, \xi\right)\right\}}{\xi-h_{0}} \geqq \frac{1}{2} .
$$

Then we have

$$
\begin{aligned}
\frac{\mu\left\{G \cap\left(h_{0}, x\right)\right\}}{x-h_{0}} & =\frac{\mu\left\{G \cap\left(h_{0}, \xi\right)\right\}+\mu\{G \cap(\xi, x)\}}{x-h_{0}} \\
& \geqq \frac{\frac{1}{2}\left(\xi-h_{0}\right)+\frac{1}{2}(x-\xi)}{x-h_{0}}=\frac{1}{2} .
\end{aligned}
$$

This contradicts $\left(2^{\prime} 6\right)$, and we conclude that

$$
\eta=f(\xi) \text {. }
$$

Finally we show that $\xi \in E$. In fact,

$$
\underline{f}_{\mathrm{ap}}^{+}(\xi)=\liminf _{x \rightarrow \xi_{+}} \frac{f(x)-f(\xi)}{x-\xi} .
$$

Using $\left(2^{\prime} 7\right)$ we observe that

$$
\left\{x: x>\xi, \frac{f(x)-f(\xi)}{x-\xi}>0\right\} \subseteq G .
$$

Since $G$ has no unit right-density at $\xi$ (by the above argument), neither has the set on r.h.s. of $\left(2^{\prime} 9\right)$. Now $\left(2^{\prime} 8\right)$ shows immediately that $f_{\text {ap }}^{+}(\xi) \leqq 0$, i.e. $\xi \in E$. We have arrived at the final contradiction, and the proof of the theorem is complete.

3. Theorem 3'1. Suppose that (i) $f$ is a.u.s.c. on the left everywhere in $I_{0}$, and

(ii) $f_{\mathrm{ap}}^{+}(x) \geqq 0$ for all $x$ in $I_{0}$. Then $f$ is monotone increasing in $I_{0}$.

Proof. Let $x_{1}$ and $x_{2}$ be two points in $I_{0}$ with $x_{1}<x_{2}$. We shall show that $f\left(x_{2}\right) \geqq f\left(x_{1}\right)$. Without loss of generality we assume that $x_{1}=a$ and $x_{2}=b$. Let $\epsilon$, with $\epsilon>0$, be given. Consider the set $G^{*}$ $=\{x: f(x)-f(a) \geqq-\epsilon(x-a)\}$. Now we construct the point $\xi^{*}$ with the help of the set $G^{*}$ exactly in the same way as we construct the point $\xi$ in Theorem $2^{\prime} 1$. It is also established as in Theorem 2'1 that $G^{*}$ has no zero left-density at $\xi^{*}$. Since $f$ is a.u.s.c on the left at $\xi^{*}$, it follows easily that $\xi^{*} \in G^{*}$. Clearly $\xi^{*} \leqq b$. Suppose that $\xi^{*}<b$. Since $\underline{f}_{\text {ap }}^{+}(\xi) \geqq 0$, we can find $x \in G^{*}$, with $x>\xi^{*}$, such that

$$
\frac{\mu\left\{G^{*} \cap\left(\xi^{*}, x\right)\right\}}{x-\xi^{*}} \geqq \frac{1}{2} .
$$


Now we offer a contradiction argument similar to that which has been used to established $\left(2^{\prime} 7\right)$ in Theorem $2^{\prime} 1$. We thus conclude that $\xi^{*}=b$. This gives, since $\xi^{*} \in G^{*}, f(b)-f(a) \geqq-\epsilon(b-a)$. Since $\epsilon$ is arbitrary, we have $f(b) \geqq f(a)$. This completes the proof. The following theorem is a generalization of Theorem $3^{\prime} 1$.

THeOREM $3^{\prime} 2$. If (i) $f$ is a.u.s.c on the left everywhere in $I_{0}$,

(ii) $f_{\mathrm{ap}}^{+}(x) \geqq 0$ almost everywhere in $I_{0}$, and $I_{0}$.

(iii) $f_{\mathrm{ap}}^{+}(x)>-\infty$ everywhere in $I_{0}$, then $f$ is monotone increasing in

Proof. Let $E=\left\{x: f_{\text {ap }}^{+}(x)<0\right\}$. By hypothesis (ii) $\mu(E)=0$. By a theorem $\left[4\right.$, p. 214] there is a continuous increasing function $\sigma$ in $I_{0}$ such that $\sigma^{\prime}(x)=+\infty$ for $x \in E$. Let $\epsilon$, with $\epsilon>0$, be given. Consider the function $\psi$ defined on $I_{0}$ by: $\psi(x)=f(x)+\epsilon \sigma(x)$. Then we have the following:

(i) $\psi$ is a.u.s.c on the left everywhere in $I_{0}$, and

(ii) $\psi_{-\mathrm{ap}}^{+}(x) \geqq f_{-\mathrm{ap}}^{+}(x)+\epsilon \sigma_{-\mathrm{ap}}^{+}(x) \geqq 0$ for all $x$ in $I_{0}$. Hence, by Theorem $3^{\prime} 1 \psi$ is monotone increasing in $I_{0}$. Since $\epsilon$ is arbitrary, we conclude that $f$ is monotone increasing in $I_{0}$. The proof is complete.

We wish to point out hypothesis (iii) in Theorem $3^{\prime} 2$ is not redundant. The following example illustrates this.

Let

$$
\begin{aligned}
f(x)=2 x & \text { if } 0 \leqq x \leqq 1 \\
& =1 \quad \text { if } 1<x \leqq 2
\end{aligned}
$$

$f$ satisfies all the conditions of Theorem $3^{\prime} 2$ except at $x=1$, where $f_{-\mathrm{ap}}^{+}(x)=-\infty . f$ is not monotone increasing in $[0,2]$.

4. Referring to Theorem $2^{\prime} 1$ we want to estimate how large the exceptional set $\left\{x: f_{-\mathrm{ap}}^{+}(x) \leqq 0\right\}$ may be without making the theorem false. In this connection we recall the following theorem.

THEOREM 4'1 [2, p. 199]. Suppose that $f$ is a measurable function in $I_{0}$. Let

(i) $S=\left\{x: f_{\mathrm{ap}}^{\prime}(x)\right.$ exists, and is finite $\}$, and

(ii) $T=\{x$ : all four approximate derivates are infinite at $x\}$. Then $\mu\left\{I_{0} \backslash(S \cup T)\right\}=0$.

As an analogue to this we propose the following theorem. 
THEOREM 4'2. Let $f$ be a measurable function on $I_{0}$ such that

(i) $E_{1}=\left\{x: f_{\mathrm{ap}}^{\prime}(x)\right.$ exists and $\left.\neq 0\right\}$, and

(ii) $E_{2}=\{x$ : all four approximate derivates are infinite at $x\}$, then $\mu\left[f\left\{I_{0} \backslash\left(E_{1} \cup E_{2}\right)\right\}\right]=0$.

We need the following lemmas.

Lemma 4'1. Let $f$ be a measurable function, and $\lambda$ be a real number. Let $E=\left\{x: f_{\mathrm{ap}}^{\prime}(x)=\lambda\right\}$. Then

$$
\mu\{f(E)\} \leqq|\lambda| \mu(E) .
$$

Proof. By a theorem of S. Saks [3, p. 239], we can write $E=\cup_{n=1}^{\infty} E_{n}$ where $f$ is absolutely continuous on each $E_{n}(n=1,2, \cdots)$. The sets $E_{n}$ may be taken to be pairwise disjoint. Since $f$ is absolutely continuous on $E_{n}$, it satisfies Lusin's $(N)$-condition on $E_{n}$ [3, p. 225], and it is of bounded variation on $E_{n}(n=1,2, \cdots)$. Therefore, by a lemma of S. Saks $\left[3\right.$, p. 221] there is a function $g_{n}$ which is of bounded variation in $I_{0}$ such that $f(x)=g_{n}(x)$ for $x \in E_{n}$. Clearly, $g_{n}^{\prime}(x)$ $=f_{\mathrm{ap}}^{\prime}(x)=\lambda$ whenever $x \in E_{n} \backslash B_{n}$, where $B_{n}$ is a subset of $E_{n}$ with $\mu\left(B_{n}\right)=0$. Since $f$ satisfies Lusin's $(N)$-condition over $E_{n}$, we have

$$
\mu\left\{f\left(B_{n}\right)\right\}=0 .
$$

Using a known result $[3$, p. 227] we have

$$
\mu\left\{f\left(E_{n} \backslash B_{n}\right)\right\}=\mu\left\{g_{n}\left(E_{n} \backslash B_{n}\right)\right\} \leqq \int_{E_{n} \backslash B_{n}}\left|g_{n}^{\prime}(x)\right| d x=|\lambda| \mu\left(E_{n} \backslash B_{n}\right) .
$$

From (4'1) we deduce $\mu\left\{f\left(E_{n}\right)\right\} \leqq|\lambda| \mu\left(E_{n}\right)$. Since $E=\cup_{n=1}^{\infty} E_{n}$, and $E_{n}$ are pairwise disjoint, we have

$$
\mu\{f(E)\} \leqq \sum_{n=1}^{\infty} \mu\left\{f\left(E_{n}\right)\right\} \leqq|\lambda| \sum_{n=1}^{\infty} \mu\left(E_{n}\right)=|\lambda| \mu(E) .
$$

Lemma 4'2. Let $f$ be a measurable function on $I_{0}$ and let $E^{\prime}=\{x:$ at least one approximate derivate is finite at $x\}$. Then $f$ satisfies Lusin's $(N)$-condition on $E^{\prime}$.

This lemma is in S. Saks [3, pp. 290-292].

Proof of Theorem $4^{\prime} 2$. Let $E_{3}=\left\{x: f_{\text {ap }}^{\prime}(x)\right.$ exists, and $\left.=0\right\}$, and $E_{4}=\left\{x: f_{\mathrm{ap}}^{\prime}(x)\right.$ does not exist, and at least one of the four approximate derivates is finite at $x\}$. Clearly $I_{0} \backslash\left(E_{1} \cup E_{2}\right) \subseteq E_{3} \cup E_{4}$. Hence, 


$$
\mu\left[f\left\{I_{0} \backslash\left(E_{1} \cup E_{2}\right)\right] \leqq \mu\left\{f\left(E_{3}\right)\right\}+\mu\left\{f\left(E_{4}\right)\right\} .\right.
$$

By Lemma $4^{\prime} 1$

$$
\mu\left\{f\left(E_{3}\right)\right\}=0 .
$$

According to Theorem 4'1 $E_{4} \subseteq I_{0} \backslash(S \cup T)$, and hence $\mu\left(E_{4}\right)=0$. Using Lemma $4^{\prime} 2$ we have

$$
\mu\left\{f\left(E_{4}\right)\right\}=0 .
$$

From $\left(4^{\prime} 2\right),\left(4^{\prime} 3\right)$ and $\left(4^{\prime} 4\right)$ we have $\mu\left[f\left\{I_{0} \backslash\left(E_{1} \cup E_{2}\right)\right\}\right]=0$.

Theorem 4'3. Suppose that $f$ is a measurable function on $I_{0}$. Let (i) $f$ be a.u.s.c. on the left and a.l.s.c. on the right everywhere in $I_{0}$,

(ii) $P=\left\{x:-\infty<f_{\mathrm{ap}}^{\prime}(x)<0\right\}$, and $\mu(P)=0$, and

(iii) $Q=\left\{x\right.$ : all four approximate derivates are infinite with $f_{\text {ap }}^{+}(x)$ $=-\infty$, and $Q$ be countable $\}$. Then $f$ is monotone increasing in $I_{0}$.

Proof. Let $H=\left\{x: f_{-a p}^{+}(x) \leqq 0\right\}$. Then we have

$$
\begin{aligned}
H \backslash\left\{I_{0} \backslash\left(E_{1} \cup E_{2}\right)\right\} & =H \cap\left(E_{1} \cup E_{2}\right) \\
& =\left(H \cap E_{1}\right) \cup\left(H \cap E_{2}\right) \subset P \cup Q .
\end{aligned}
$$

Now

$$
\begin{aligned}
\mu\{f(H)\} & \leqq \mu\left[f\left\{H \backslash\left(I_{0} \backslash\left(E_{1} \cup E_{2}\right)\right)\right\}\right]+\mu\left[f\left\{I_{0} \backslash\left(E_{1} \cup E_{2}\right)\right\}\right] \\
& =\mu\left[f\left\{H \backslash\left(I_{0} \backslash\left(E_{1} \cup E_{2}\right)\right)\right\}\right]
\end{aligned}
$$

by Theorem $4^{\prime} 2$. Thus

$$
\mu\{f(H)\} \leqq \mu\{f(P)\}+\mu\{f(Q)\} .
$$

By hypothesis (ii), $\mu(P)=0$ and by Lemma $4^{\prime} 2$

$$
\mu\{f(P)\}=0 .
$$

Let us suppose that $\mu\{f(Q)\}>0$. Then the cardinality of $Q$ must be equal to that of the continuum, and hence $Q$ is uncountable. This contradicts hypothesis (iii). Hence we conclude that

$$
\mu\{f(Q)\}=0 .
$$

From $\left(4^{\prime} 5\right),\left(4^{\prime} 6\right)$ and $\left(4^{\prime} 7\right)$ we obtain $\mu\{f(H)\}=0$. Therefore Int $\{f(H)\}=\varnothing$. An application of Theorem $2^{\prime} 1$ now completes the proof. 


\section{REFERENCES}

1. C. Goffman and C. J. Neugebauer, On approximate derivatives, Proc. Amer. Math. Soc. 11 (1960), 962-966.

2. R. L. Jeffery, The theory of functions of a real variable, Math. Exposition, no. 6, Univ. of Toronto Press, Ontario, 1951.

3. S. Saks, Theory of integrals, Warszawa-Lwow, 1937.

4. I. P. Natanson, Theory of functions of a real variable. Vol. I, Constable \& Co.

BURDWAN UNIVERSITy, INDIA 